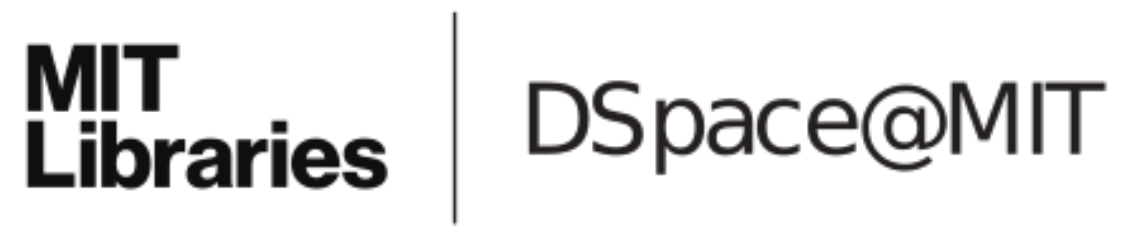

\author{
MIT Open Access Articles
}

\section{Fundamental Bounds on First Passage \\ Time Fluctuations for Currents}

The MIT Faculty has made this article openly available. Please share how this access benefits you. Your story matters.

Citation: Gingrich, Todd R. and Horowitz, Jordan M. "Fundamental Bounds on First Passage Time Fluctuations for Currents." Physical Review Letters 119, 17 (October 2017): 170601 (c) 2017 American Physical Society

As Published: http://dx.doi.org/10.1103/PhysRevLett.119.170601

Publisher: American Physical Society

Persistent URL: http://hdl.handle.net/1721.1/112979

Version: Final published version: final published article, as it appeared in a journal, conference proceedings, or other formally published context

Terms of Use: Article is made available in accordance with the publisher's policy and may be subject to US copyright law. Please refer to the publisher's site for terms of use. 


\title{
Fundamental Bounds on First Passage Time Fluctuations for Currents
}

\author{
Todd R. Gingrich* and Jordan M. Horowitz \\ Physics of Living Systems Group, Department of Physics, Massachusetts Institute of Technology, \\ 400 Technology Square, Cambridge, Massachusetts 02139, USA
}

(Received 26 June 2017; revised manuscript received 10 August 2017; published 24 October 2017)

\begin{abstract}
Current is a characteristic feature of nonequilibrium systems. In stochastic systems, these currents exhibit fluctuations constrained by the rate of dissipation in accordance with the recently discovered thermodynamic uncertainty relation. Here, we derive a conjugate uncertainty relationship for the first passage time to accumulate a fixed net current. More generally, we use the tools of large-deviation theory to simply connect current fluctuations and first passage time fluctuations in the limit of long times and large currents. With this connection, previously discovered symmetries and bounds on the large-deviation function for currents are readily transferred to first passage times.
\end{abstract}

DOI: 10.1103/PhysRevLett.119.170601

Introduction.-Thermodynamics constrains the fluctuations of nonequilibrium systems, as evidenced by a growing collection of universal predictions connecting dissipation to fluctuations. Examples include the fluctuation theorems [1-7], nonequilibrium fluctuation-dissipation theorems [8-14], and, more recently, the thermodynamic uncertainty relation [15-17]. Remarkably, all these results can be viewed through one unifying lens, namely, large-deviation theory [18]. In fact, over the past two decades this formalism has proven to be an essential tool for characterizing the dynamical fluctuations of nonequilibrium systems [19-26].

Recently, these techniques have revealed a universal inequality between the far-from-equilibrium fluctuations in current-such as the flow of particles, energy, or entropy - with the near-equilibrium fluctuations predicted by linear-response theory [16]. A useful corollary is the thermodynamic uncertainty relation [15], which offers a fundamental trade-off between typical current fluctuations and dissipation [27]. Specifically, a nonequilibrium Markov process generating an average time-integrated current $\langle J\rangle$ during a long observation time $T_{\text {obs }}$ has a variance $\operatorname{Var}(J)$ constrained by the mean entropy-production rate $\sigma$ (with Boltzmann's constant $k_{B}=1$ ):

$$
\frac{\operatorname{Var}(J)}{\langle J\rangle^{2}} \geq \frac{2}{T_{\text {obs }} \sigma}
$$

Thus, reducing fluctuations comes with an energetic cost.

A significant body of recent work has analyzed such current fluctuations for a fixed observation time $[15,16$, 28-35]. In this Letter, we consider the complementary problem, analyzing the fluctuations of first passage times $T$ to reach a large threshold time-integrated current $J_{\text {thr }}$ (see Fig. 1). We show that properties of the first passage time distribution for asymptotically large $J_{\text {thr }}$ follow simply from knowledge of the current fluctuations. This conjugate relationship between fixed-time and fixed-current trajectory ensembles mirrors the study of inverse or adjoint processes in queuing theory [36-38], and it extends Garrahan's work on first passage time fluctuations of dynamical activity-a monotonically increasing counting variable [39,40]- to current variables, which can grow or shrink. By relating the conjugate problems, we are able to transform inequalities governing current fluctuations into associated inequalities for passage time fluctuations, as well as offer fresh insight into recent predictions for entropy-production first passage times [41-44]. For instance, we show that the distribution for the time $T$ to first hit a large threshold current $J_{\text {thr }}$ must satisfy a corresponding uncertainty relation:

$$
\frac{\operatorname{Var}(T)}{\langle T\rangle^{2}} \geq \frac{2}{\langle T\rangle \sigma}
$$

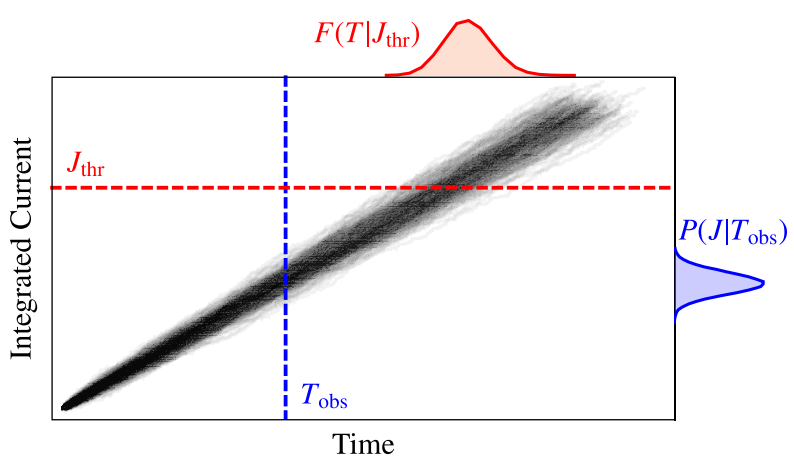

FIG. 1. The distribution of integrated current $J$ for a long observation time $T_{\text {obs }}$ and the distribution for first passage time $T$ to a large threshold current $J_{\text {thr }}$ are two faces of the same distribution over trajectories. Hence, recent results describing the asymptotic form of the current distribution $P\left(J \mid T_{\text {obs }}\right)$ naturally yield corresponding results for the asymptotic form of the first passage time distribution $F\left(T \mid J_{\mathrm{thr}}\right)$. 
The two faces of the thermodynamic uncertainty relationship can be viewed as two ways to infer a bound on the entropy-production rate-one utilizing the current fluctuations in a fixed-time ensemble and the other utilizing the time fluctuations in a fixed-current ensemble. Though these two sets of fluctuations contain equivalent information, we emphasize that the physical measurements are quite distinct.

Setup.-To make the notions concrete, we focus our presentation on nonequilibrium systems that can be modeled as Markov jump processes. Specifically, we have in mind a mesoscopic system with states $i=1, \ldots, N$, whose time-varying probability density $\boldsymbol{p}=\left\{p_{i}\right\}_{i=1}^{N}$ evolves according to the master equation $\dot{\boldsymbol{p}}=\mathbb{W} \boldsymbol{p}$, where $\mathbb{W}_{i j}$ is the probability rate to transition from $j \rightarrow i$, and $-\mathbb{W}_{i i}=$ $\sum_{j \neq i} \mathbb{W}_{j i}$ is the exit rate from $i$. We assume that $\mathbb{W}$ is irreducible — so that a unique steady-state exists—and that every transition is reversible, that is, $\mathbb{W}_{i j} \neq 0$ only when $\mathbb{W}_{j i} \neq 0$. Thermodynamics enters by requiring transitions to satisfy local detailed balance. The ratio of rates for each transition can then be identified with a generalized thermodynamic force $\mathcal{F}_{i j}=\ln \left(\mathbb{W}_{i j} / \mathbb{W}_{j i}\right)$ [45], which quantifies the flow of free energy into the surrounding environment [46].

Fluctuating currents represent the net buildup of transitions between the system's mesoscopic states. Indeed, in any given stochastic realization of our system's evolution, there will be some random number of net transitions, or current, between every pair of states $j \rightarrow i$, which we label as $J_{i j}$. Our interest though is in generalized currents obtained as superpositions of mesoscopic transitions, $J \equiv \sum_{i>j} d_{i j} J_{i j}$, where the $d_{i j}$ indicate how much a particular transition contributes. Such generalized currents often represent a measurable global flow through the system, such as the ATP consumption throughout a biochemical network or the net flow of heat between multiple thermal reservoirs [46]. A particularly important example is the fluctuating environmental entropy production $\Sigma$ obtained by choosing $d_{i j}=\ln \mathbb{W}_{i j} / \mathbb{W}_{j i}$. Its average rate $\sigma=\lim _{T_{\text {obs }} \rightarrow \infty}\langle\Sigma\rangle / T_{\text {obs }}$ measures the time irreversibility of the dynamics.

For long observation times $T_{\mathrm{obs}}$, the probability of observing a current $J$ satisfies a large-deviation principle $P\left(J \mid T_{\mathrm{obs}}\right) \asymp e^{-T_{\mathrm{obs}} I\left(J / T_{\mathrm{obs}}\right)}$ with large-deviation rate function $I(j)$ [18], where the lowercase letter $j \equiv J / T_{\text {obs }}$ represents an intensive quantity. The large-deviation function $I$ captures not just the typical fluctuations predicted by the central-limit theorem but also the relative likelihood of exponentially rare events. A useful complementary characterization of the fluctuations is through the scaled cumulant generating function (SCGF) $\quad \psi(\lambda)=\lim _{T_{\mathrm{obs}} \rightarrow \infty}\left(1 / T_{\mathrm{obs}}\right) \ln \left\langle e^{-\lambda J}\right\rangle$, with the expectation taken over trajectories of length $T_{\text {obs }}$. Derivatives of $\psi$ at the origin encode all the long-time

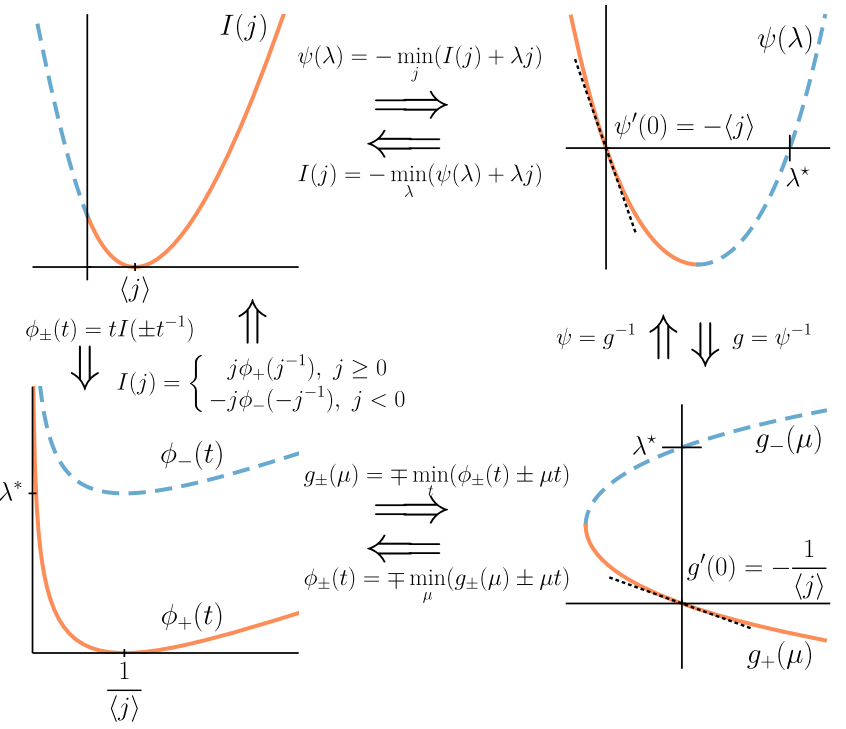

FIG. 2. Large deviation rate functions (left) are related to SCGFs (right) by the Legendre-Fenchel transform. Current statistics (top) and first passage time statistics (bottom) are connected by inversion. Branches corresponding to positive currents are plotted with solid red lines, while the negativecurrent branches are plotted with dashed blue lines.

current cumulants. The pair $I$ and $\psi$ are intimately related through the Legendre-Fenchel transform, as graphically illustrated in Fig. 2 [18].

Universal symmetries and bounds on I (commensurately $\psi$ ) have refined our understanding of the thermodynamics of nonequilibrium systems. In the following, we develop a complementary point of view based on current first passage times.

First passage time fluctuations for large current.-We now consider a large (in magnitude) fixed amount of accumulated current $J_{\text {thr }}$ and seek the time at which that threshold current is first reached. As seen in Fig. 1, the mean first passage time scales extensively with the magnitude of $J_{\text {thr }}$, suggesting a large-deviation form for the first passage time distribution $F\left(T \mid J_{\text {thr }}\right)$. We note, however, that $J_{\text {thr }}$ can be either positive or negative and introduce two different rate functions, $\phi_{+}(t)$ and $\phi_{-}(t)$, to handle these cases:

$$
F\left(T \mid J_{\text {thr }}\right) \asymp \begin{cases}e^{-J_{\text {thr }} \phi_{+}\left(T / J_{\text {thr }}\right)}, & J_{\text {thr }}>0, \\ e^{J_{\text {thr }} \phi_{-}\left(-T / J_{\text {thr }}\right)}, & J_{\text {thr }}<0 .\end{cases}
$$

Correspondingly, there are now two different SCGFs $g_{ \pm}(\mu)=\lim _{J_{\text {thr }} \rightarrow \pm \infty}\left(1 / J_{\text {thr }}\right) \ln \left\langle e^{-\mu T}\right\rangle$, with the expectation computed over trajectories having a fixed value $J_{\text {thr }}$ of timeintegrated current. Without loss of generality, we assume a choice of $\left\{d_{i j}\right\}$ such that $\langle J\rangle>0$. In this case, the + subscript corresponds to branches quantifying typical (positivecurrent) fluctuations, and the - subscript corresponds to rare 
(negative-current) branches. It is useful to also split $\psi$ into two branches, $\psi_{+}$with negative slope and $\psi_{-}$with positive slope (see Fig. 2). Our central result is that the large deviations in scaled first passage times $t \equiv T /\left|J_{\text {thr }}\right|$ are completely determined by the large-deviation functions for current fluctuations:

$$
\phi_{ \pm}(t)=t I( \pm 1 / t), \quad g_{ \pm}(\mu)=\psi_{ \pm}^{-1}(\mu) .
$$

Analogous relations have appeared for counting variables [36-38,40] and for entropy-production fluctuations [42], but we show these connections are, in fact, more general and extend to all currents. Thus, all known properties of $I$ - most notably, symmetries and bounds-can naturally be translated to $\phi$.

Here, we offer a heuristic argument for Eq. (4) assuming positive current. A sketch of a proof is included at the end of the Letter, and a more detailed proof is provided in the Supplemental Material [47]. To start, we write $\mathcal{P}(\gamma)$ to denote the probability distribution for a mesoscopic trajectory $\gamma$-that is, a sequence of states visited by the system and their jump times. Then, the likelihood of a large first passage time $T=t J$ to a large current $J$ can be conveniently expressed as

$$
P(T=t J)=\int d \gamma \delta(T-t J) \mathcal{P}(\gamma),
$$

where the integral is over all trajectories. However, the only trajectories that can contribute to this integral have current $J$. Furthermore, large current can only be attained after a long time. Taken together, these observations suggest we can replace $\mathcal{P}$ with the large-deviation form for large $T$ [48]:

$P(T=t J) \asymp \int d J \delta(T-t J) e^{-T I(J / T)}=e^{-J t I(1 / t)}$,

which implies $\phi_{+}(t)=t I(1 / t)$, and $g_{+}(\mu)$ follows by the Legendre-Fenchel transform. Put simply, switching from current to first passage time is a change of variables where we replace current by its inverse.

We now turn to the implications of Eq. (4). For any generalized current, its long-time fluctuations are constrained by the entropy-production rate via Eq. (1). This constraint actually follows from an inequality on the largedeviation rate function,

$$
I(j) \leq \frac{(j-\langle j\rangle)^{2}}{4\langle j\rangle^{2}} \sigma \equiv I_{\text {bnd }}(j) .
$$

Translating to first passage time fluctuations, we have

$$
\phi_{+}(t) \leq \frac{(t-\langle t\rangle)^{2}}{4 t} \sigma \equiv \phi_{\text {bnd }}(t),
$$

after noting that the typical behavior $\langle j\rangle=1 /\langle t\rangle$ does not depend on the choice of ensemble-fixed $T_{\text {obs }}$ versus fixed $J_{\mathrm{thr}}$. Equation (2) follows since the large $J_{\mathrm{thr}}$ variance is computed in terms of derivatives of the large-deviation function as $\operatorname{Var}(T)=J_{\text {thr }} / \phi_{+}^{\prime \prime}(\langle t\rangle)$ [18]. Thus, dissipation is a fundamental constraint to controlling first passage time fluctuations as well as current fluctuations.

Together, Eqs. (7) and (8) point to a remarkable property of the stochastic evolution of currents, which is best appreciated by normalizing the large-deviation forms $e^{-T_{\text {obs }} I_{\text {bnd }}(j)}$ and $e^{-J_{\text {thr }} \phi_{\text {bnd }}(t)}$. For currents, we have a Gaussian distribution

$$
P_{\text {bnd }}(j)=\sqrt{\frac{T_{\text {obs }} \sigma}{4 \pi\langle j\rangle^{2}}} \exp \left(-\frac{T_{\text {obs }}(j-\langle j\rangle)^{2} \sigma}{4\langle j\rangle^{2}}\right),
$$

whereas the first passage time distribution is an inverse Gaussian

$$
F_{\text {bnd }}(t)=\sqrt{\frac{J_{\text {thr }} \sigma\langle t\rangle^{2}}{4 \pi t^{3}}} \exp \left(-\frac{J_{\text {thr }}(t-\langle t\rangle)^{2} \sigma}{4 t}\right) .
$$

Remarkably, these are the distributions we would have predicted if we had simply treated the evolution of the current as a one-dimensional diffusion process with constant drift $\langle j\rangle$ and diffusion coefficient $\sigma /\langle j\rangle^{2}$ [49]. This observation suggests that while the precise dynamics of the currents is generally complex, there is a simple auxiliary diffusion process that constrains it, reminiscent of the universal form observed for the stochastic evolution of the entropy production as a drift-diffusion process $[7,44]$.

First passage time fluctuations for negative current and the fluctuation theorem.-We have focused primarily on first passage times to reach a (typical) positive current. We can also consider the first passage time to the exponentially suppressed negative currents that arise due to trajectories that appear to run backwards in time. The distribution for the time to reach $J_{\mathrm{thr}}<0$ scales according to $\phi_{-}(t)$, which can be related to $\psi_{-}(\lambda)$ (see Fig. 2). This connection is especially interesting when $\psi$ posses a symmetry that relates its two branches $\psi_{+}$and $\psi_{-}$, because this naturally translates to a relationship between $\phi_{+}$and $\phi_{-}$.

Generically, $\psi_{-}$vanishes at some $\lambda^{*}$. For certain currents, it also satisfies $\psi_{+}(\lambda)=\psi_{-}\left(\lambda^{*}-\lambda\right)$. As an example, the fluctuation theorem implies such a symmetry with $\lambda^{*}=1$ for the entropy production (itself a generalized current) [6]. Symmetry of $\psi$ yields a corresponding symmetry in $g_{ \pm}$: $g_{+}(\mu)=-g_{-}(\mu)+\lambda^{*}$. Taking the Legendre-Fenchel transform gives

$$
\phi_{+}(t)=\phi_{-}(t)-\lambda^{*},
$$

indicating that $\phi_{+}$and $\phi_{-}$differ by a constant offset when the SCGF symmetry is present. Equation (11) must be 
interpreted carefully, as it compares large-deviation functions for two different distributions. Typically, largedeviation rate functions are shifted such that their minimum equals zero. In this case, a symmetrical $\psi$ implies that $\phi_{+}$ and $\phi_{-}$are identical, and the large-current first passage time distribution $F\left(T \mid J_{\text {thr }}\right)$ is the same for both positive and negative $J_{\text {thr }}$. While the constant offset in Eq. (11) does not affect the form of $F\left(T \mid J_{\text {thr }}\right)$, it reflects the fact that the probability of reaching $\left|J_{\mathrm{thr}}\right|$ exceeds that of reaching $-\left|J_{\text {thr }}\right|$ by a factor of $e^{\lambda^{*}\left|J_{\text {thr }}\right|}$. Using the same methods as those in this Letter, Saito and Dhar reached similar conclusions for the case that the generalized current is the entropy production [42], and Neri et al. have proven a corresponding fluctuation theorem for entropy-production stopping times using Martingale theory [43]. Our result, Eq. (11), extends more generally to any current satisfying a SCGF symmetry about $\lambda^{*}$, including the example of the next section.

Illustrative example.-To demonstrate the bounds in a more explicit context, we solve for the large-deviation behavior of a minimal model for an enzyme-mediated reaction from reactant $R$ to product $P$. The enzyme can be either in a ground state $E$ or an activated state $E^{*}$, and the $E \leftrightarrow E^{*}$ transformations proceed via one of three pathways: (1) the enzyme exchanges heat with a thermal bath, (2) the enzyme accepts free energy by converting an activated fuel molecule $F^{*}$ into a deactivated form $F$, or (3) the activated enzyme converts $R \rightarrow P$. Each of these pathways proceeds forward or backward, as depicted in Fig. 3, with six rate constants defining the model. We follow the net transformations of $R$ into $P$ as the accumulated current $J$, so the first passage time can be interpreted as the time to generate $J$ product molecules.

The analytical solution of this model using standard methods is outlined in the Supplemental Material [47]. Figure 3 graphically shows the large-deviation function

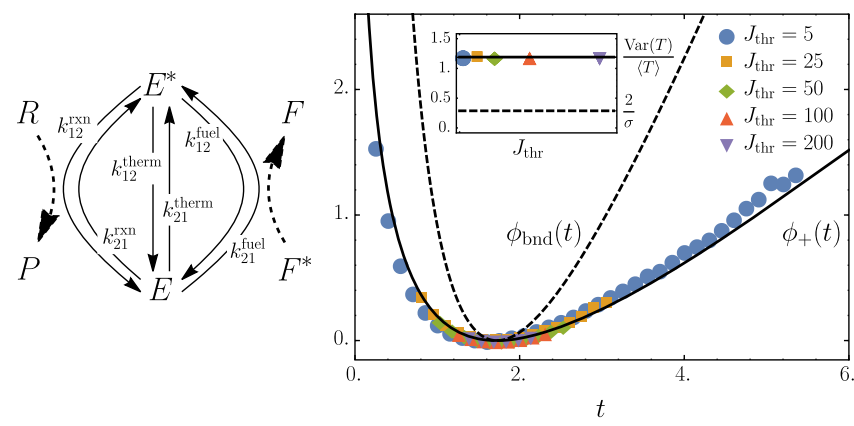

FIG. 3. Markov model for the conversion of a reactant $R$ to product $P$ mediated by enzyme $E$. The large deviation function for the time to reach a particular net current from $R$ to $P, \phi_{+}(t)$, is bounded by $\phi_{\text {bnd }}(t)$. Additionally, $\phi_{+}(t)$ is inferred from numerical sampling of $10^{6}$ trajectories for various choices of $J_{\text {thr }}$ using rates $k_{12}^{\mathrm{rxn}}=2, k_{21}^{\mathrm{rxn}}=0.1, k_{12}^{\text {therm }}=0.3, k_{21}^{\text {therm }}=0.001$, $k_{12}^{\text {fuel }}=0.001, k_{21}^{\text {fuel }}=1$. bound, Eq. (8), as well as the uncertainty bound, Eq. (2) (see inset). The analytical calculations are supplemented by trajectory sampling with finite $J_{\text {thr }}$, the results of which are plotted with colored markers in Fig. 3. Motivated by the $t^{-3 / 2}$ prefactor in Eq. (10), we extract estimates for $\phi_{+}(t)$ from the sampled trajectories by first approximating $F\left(T \mid J_{\text {thr }}\right)$ with a histogram and then computing

$$
\phi_{+}^{\text {est }}(t)=-\frac{1}{J_{\mathrm{thr}}}\left(\ln F\left(t J_{\mathrm{thr}} \mid J_{\mathrm{thr}}\right)+\frac{3}{2} \ln t\right)+C_{\mathrm{off}},
$$

where $C_{\text {off }}$ is a constant offset used to set the minimum of $\phi_{+}^{\text {est }}$ to zero. We observe that the large-deviation form (and, consequently, the thermodynamic uncertainty relation) remain valid even for small $J_{\mathrm{thr}}$.

Conclusion.-In the large-deviation limit, we have shown that current fluctuations with fixed observation time are intimately related to the fluctuations in first passage times to large current. As a result, we have seen how the thermodynamic uncertainty relation and the fluctuation theorem for entropy production naturally lead to a universal symmetry and bounds on first passage time fluctuations. Tighter-than-quadratic bounds on current large-deviation fluctuations $[28,30,31]$ also readily translate to corresponding first passage time bounds.

Practically, we anticipate that it will be useful to convert between fixed-time and fixed-current ensembles since some experiments are more naturally suited to one than the other. For example, imagine we seek a dissipation bound for the enzyme-mediated reaction in Fig. 3. Fluctuations in product formation after time $T_{\text {obs }}$ could be measured spectroscopically, assuming Beer's law and a calibrated mapping from fluorescence intensity to product concentration. But the fixed $J_{\text {thr }}$ ensemble offers an advantage. By measuring first passage time fluctuations to reach a fixed fluorescence intensity, the mapping between fluorescence and concentration could be avoided altogether. More ambitiously, we expect the fluctuating time ensemble to be a natural way to analyze the role of dissipation in Brownian clocks [50-54].

Sketch of a proof for Eq. (4). - The main result, Eq. (4), consists of two relations: one connects the large-deviation rate function $I$ with $\phi_{ \pm}$, the other connects $\psi$ with $g$. Here we sketch a proof of $g_{ \pm}(\mu)=\psi_{ \pm}^{-1}(\mu)$. The relationship between $I$ and $\phi_{ \pm}$follows by applying the Gärtner-Ellis theorem to compute $I$ from $\psi$ and $\phi_{ \pm}$from $g_{ \pm}$. More details are presented in the Supplemental Material [47].

The basic strategy is to express both $g$ and $\psi$ in terms of spectral properties of a tilted rate matrix $\mathbb{W}(\lambda)$, whose elements are given by $\mathbb{W}_{i j}(\lambda)=\mathbb{W}_{i j} e^{-\lambda d_{i j}}$. The first half of this connection is well known; the largest eigenvalue of $\mathbb{W}(\lambda)$ is the SCGF $\psi(\lambda)$ [6]. Expressing $g$ in terms of the tilted rate matrix requires a slightly more involved calculation following the general strategy of Refs. [40,42]. 
Let $F_{i j}(T \mid J)$ be the distribution of times $T$ to first accumulate $J$ current with a jump to $i$, conditioned upon a start in $j$. We connect $F_{i j}$ to the transition probability $P_{i j}(J, T)$ to go from $j \rightarrow i$ in time $T$, having accumulated current $J$ via the renewal equation: $\mathbf{P}(J, T)=$ $\int_{0}^{T} d t \mathbf{P}(0, T-t) \cdot \mathbf{F}(t \mid J)$ written in matrix notation. The convolution is simplified by Laplace transform (denoted with a tilde) to convert from $T$ to $\mu$, ultimately yielding $e^{-J g_{ \pm}(\mu)} \asymp\left\langle e^{-\mu T}\right\rangle=\tilde{F}(\mu \mid J) \asymp \tilde{\mathbf{P}}(J, \mu)$. Furthermore, $\tilde{\mathbf{P}}(J, \mu)$ can be expressed in terms of the tilted rate matrix via an inverse Laplace transform of $\hat{\tilde{\mathbf{P}}}(\lambda, \mu)=$ $1 /(\mathbb{W}(\lambda)-\mu \llbracket)$, where the caret denotes a Laplace transform from $J$ to $\lambda$. Using complex analysis to perform the inverse transform, we obtain $e^{-J g_{ \pm}(\mu)} \asymp e^{\bar{\lambda} J}$, where $\bar{\lambda}=$ $\psi_{+}^{-1}(\mu)$ for $J>0$ and $\bar{\lambda}=\psi_{-}^{-1}(\mu)$ for $J<0$. Hence, $g_{ \pm}$and $\psi_{ \pm}$are inverses.

We gratefully acknowledge the Gordon and Betty Moore Foundation for supporting T. R. G. and J. M. H. as Physics of Living Systems Fellows through Grant No. GBMF4513.

*toddging@mit.edu

[1] D. J. Evans and D. J. Searles, Equilibrium microstates which generate second law violating steady states, Phys. Rev. E 50, 1645 (1994).

[2] G. Gallavotti and E. G. D. Cohen, Dynamical Ensembles in Nonequilibrium Statistical Mechanics, Phys. Rev. Lett. 74, 2694 (1995).

[3] C. Jarzynski, Nonequilibrium Equality for Free Energy Differences, Phys. Rev. Lett. 78, 2690 (1997).

[4] J. Kurchan, Fluctuation theorem for stochastic dynamics, J. Phys. A 31, 3719 (1998).

[5] G. E. Crooks, Entropy production fluctuation theorem and the nonequilibrium work relation for free energy differences, Phys. Rev. E 60, 2721 (1999).

[6] J. L. Lebowitz and H. Spohn, A Gallavotti-Cohen-Type Symmetry in the Large Deviation Functional for Stochastic Dynamics, J. Stat. Phys. 95, 333 (1999).

[7] U. Seifert, Entropy Production along a Stochastic Trajectory and an Integral Fluctuation Theorem, Phys. Rev. Lett. 95, 040602 (2005).

[8] T. Speck and U. Seifert, Restoring a fluctuation-dissipation theorem in a nonequilibrium steady state, Europhys. Lett. 74, 391 (2006).

[9] J. Prost, J. F. Joanny, and J. M. R. Parrondo, Generalized Fluctuation-Dissipation Theorem for Steady-State Systems, Phys. Rev. Lett. 103, 090601 (2009).

[10] M. Baiesi, C. Maes, and B. Wynants, Fluctuations and Response of Nonequilibrium States, Phys. Rev. Lett. 103, 010602 (2009).

[11] U. Seifert and T. Speck, Fluctuation-dissipation theorem in nonequilibrium steady states, Europhys. Lett. 89, 10007 (2010).

[12] R. Chetrite and S. Gupta, Two Refreshing Views of Fluctuation Theorems Through Kinematics Elements and Exponential Martingale, J. Stat. Phys. 143, 543 (2011).
[13] M. Baiesi and C. Maes, An update on the nonequilibrium linear response, New J. Phys. 15, 013004 (2013).

[14] C. Maes, On the Second Fluctuation-Dissipation Theorem for Nonequilibrium Baths, J. Stat. Phys. 154, 705 (2014).

[15] A. C. Barato and U. Seifert, Thermodynamic Uncertainty Relation for Biomolecular Processes, Phys. Rev. Lett. 114, 158101 (2015).

[16] T. R. Gingrich, J. M. Horowitz, N. Perunov, and J. L. England, Dissipation Bounds All Steady-State Current Fluctuations, Phys. Rev. Lett. 116, 120601 (2016).

[17] C. Maes, Frenetic bounds on the entropy production, arXiv:1705.07412.

[18] H. Touchette, The large deviation approach to statistical mechanics, Phys. Rep. 478, 1 (2009).

[19] C. Maes and K. Netočný, Minimum entropy production principle from a dynamical fluctuation law, J. Math. Phys. (N.Y.) 48, 053306 (2007).

[20] C. Maes and K. Netočný, Canonical structure of dynamical fluctuations in mesoscopic nonequilibrium steady states, Europhys. Lett. 82, 30003 (2008).

[21] C. Maes, K. Netočný, and B. Wynants, On and beyond entropy production: the case of Markov jump processes, Markov Processes Relat. Fields 14, 445 (2008).

[22] D. Chandler and J. P. Garrahan, Dynamics on the way to forming glass: bubbles in space-time, Annu. Rev. Phys. Chem. 61, 191 (2010).

[23] R. Chetrite and H. Touchette, Nonequilibrium Microcanonical and Canonical Ensembles and Their Equivalence, Phys. Rev. Lett. 111, 120601 (2013).

[24] H. Touchette and R. J. Harris, Large Deviation Approach to Nonequilibrium Systems, Nonequilibrium Statistical Physics of Small Systems: Fluctuation Relations and Beyond (Wiley, Weinheim, 2013), p. 335.

[25] L. Bertini, A. Faggionato, and D. Gabrielli, Large deviations of the empirical flow for continuous time Markov chains, Ann. Inst. H. Poincaré Probab. Statist. 51, 867 (2015).

[26] L. Bertini, A. Faggionato, and D. Gabrielli, Flows, currents, and cycles for Markov chains: Large deviation asymptotics, Stoch. Proc. Appl. 125, 2786 (2015).

[27] In the long-time limit, the typical fluctuations exhibit small deviations about the steady-state current.

[28] P. Pietzonka, A. C. Barato, and U. Seifert, Universal bounds on current fluctuations, Phys. Rev. E 93, 052145 (2016).

[29] P. Pietzonka, A. C. Barato, and U. Seifert, Universal bound on the efficiency of molecular motors, J. Stat. Mech. (2016) 124004.

[30] P. Pietzonka, A. C. Barato, and U. Seifert, Affinity- and topology-dependent bound on current fluctuations, J. Phys. A 49, 34LT01 (2016).

[31] M. Polettini, A. Lazarescu, and M. Esposito, Tightening the uncertainty principle for stochastic currents, Phys. Rev. E 94, 052104 (2016).

[32] P. Tsobgni Nyawo and H. Touchette, Large deviations of the current for driven periodic diffusions, Phys. Rev. E 94, 032101 (2016).

[33] P. Pietzonka, F. Ritort, and U. Seifert, Finite-time generalization of the thermodynamic uncertainty relation, Phys. Rev. E 96, 012101 (2017). 
[34] P. Pietzonka and U. Seifert, Universal trade-off between power, efficiency and constancy in steady-state heat engines, arXiv:1705.05817.

[35] T. R. Gingrich, G. M. Rotskoff, and J. M. Horowitz, Inferring dissipation from current fluctuations, J. Phys. A 50, 184004 (2017).

[36] P. W. Glynn and W. Whitt, Large deviations behavior of counting processes and their inverses, Queueing Syst. Theory Appl. 17, 107 (1994).

[37] R. Russell, Ph.D. thesis, Trinity College, Dublin, 1997.

[38] K. Duffy and M. Rodgers-Lee, Some useful functions for functional large deviations, Stochast. Stochast. Rep. 76, 267 (2004).

[39] A. A. Budini, R. M. Turner, and J. P. Garrahan, Fluctuating observation time ensembles in the thermodynamics of trajectories, J. Stat. Mech. (2014) P03012.

[40] J. P. Garrahan, Simple bounds on fluctuations and uncertainty relations for first-passage times of counting observables, Phys. Rev. E 95, 032134 (2017).

[41] É. Roldán, I. Neri, M. Dörpinghaus, H. Meyr, and F. Jülicher, Decision Making in the Arrow of Time, Phys. Rev. Lett. 115, 250602 (2015).

[42] K. Saito and A. Dhar, Waiting for rare entropic fluctuations, Europhys. Lett. 114, 50004 (2016).

[43] I. Neri, É. Roldán, and F. Jülicher, Statistics of Infima and Stopping Times of Entropy Production and Applications to Active Molecular Processes, Phys. Rev. X 7, 011019 (2017).

[44] S. Pigolotti, I. Neri, É. Roldán, and F. Jülicher, Generic Properties of Stochastic Entropy Production, Phys. Rev. Lett. 119, 140604 (2017).

[45] The thermodynamic force may alternatively be defined in terms of the steady-state density $\pi$ as $\mathcal{F}_{i j}=\ln \left(\mathbb{W}_{i j} \pi_{j} / \mathbb{W}_{j i} \pi_{i}\right)$. These two definitions differ by the change in Shannon entropy $\ln \left(\pi_{j} / \pi_{i}\right)$ which averages to zero over a long trajectory.

[46] U. Seifert, Stochastic thermodynamics, fluctuation theorems and molecular machines, Rep. Prog. Phys. 75, 126001 (2012).

[47] See Supplemental Material at http://link.aps.org/ supplemental/10.1103/PhysRevLett.119.170601 for a proof of Eq. (4) and an analytical solution of the illustrative example.

[48] In passing from $\mathcal{P}(\gamma)$ to $e^{-T I(J / T)}$, we must recognize that $I(J / T)$ measures the asymptotic probability of a trajectory with net current $J$ in time $T$, including trajectories which have already hit $J$ at earlier times. The probability that the trajectory is making a first passage dwarfs the probability of repeated passages in the large $J$ limit.

[49] S. Karlin and H. M. Taylor, A First Course in Stochastic Processes, 2nd ed. (Academic Press, Inc., Boston, 1975).

[50] H. Qian and M. Qian, Pumped Biochemical Reactions, Nonequilibrium Circulation, and Stochastic Resonance, Phys. Rev. Lett. 84, 2271 (2000).

[51] Y. Cao, H. Wang, Q. Ouyang, and Y. Tu, The free-energy cost of accurate biochemical oscillations, Nat. Phys. 11, 772 (2015).

[52] A. C. Barato and U. Seifert, Cost and Precision of Brownian Clocks, Phys. Rev. X 6, 041053 (2016).

[53] A.C. Barato and U. Seifert, Coherence of biochemical oscillations is bounded by driving force and network topology, Phys. Rev. E 95, 062409 (2017).

[54] S. Ray and A.C. Barato, Dispersion of the time spent in a state: general expression for unicyclic model and dissipation-less precision, J. Phys. A 50, 355001 (2017). 